\title{
The complete chloroplast genome of Glycyrrhiza uralensis Fisch. isolated in Korea (Fabaceae)
}

\author{
Mi-Hee KIM ${ }^{1^{* *}}$, Suhyeon PARK ${ }^{2 * *}, J^{\prime}$ hho LEE ${ }^{3}$, Jinwook BAEK ${ }^{1}$, \\ Jongsun PARK ${ }^{2^{*}}$, Gun Woong LEE $^{1^{*}}$ \\ ${ }^{1}$ Jeonju AgroBio-Materials Institute, Jeonju 54810, Korea \\ ${ }^{2}$ InfoBoss Inc. and InfoBoss Research Center, Seoul 06278, Korea \\ ${ }^{3}$ FromBio Co. Ltd., Suwon 16681, Korea \\ (Received 27 October 2021; Revised 26 December 2021; Accepted 27 December 2021)
}

\begin{abstract}
The chloroplast genome of Glycyrrhiza uralensis Fisch was sequenced to investigate intraspecific variations on the chloroplast genome. Its length is $127,689 \mathrm{bp}$ long ( $34.3 \% \mathrm{GC}$ ratio) with atypical structure of chloroplast genome, which is congruent to those of Glycyrrhiza genus. It includes 110 genes (76 protein-coding genes, four rRNAs, and 30 tRNAs). Intronic region of $n d h A$ presented the highest nucleotide diversity based on the six G. uralenesis chloroplast genomes. A total of 150 single nucleotide polymorphisms and 10 insertion and deletion (INDEL) regions were identified from the six G. uralensis chloroplast genomes. Phylogenetic trees show that the six chloroplast genomes of G. uralensis formed the two clades, requiring additional studies to understand it.
\end{abstract}

Keywords: chloroplast genome, Fabaceae, Glycyrrhiza uralensis, intraspecific variation, nucleotide diversity, phylogenetic analysis

Glycyrrhiza uralensis Fisch. is distributed mainly in Central Asia, Mongolia, and China (Hayashi and Sudo, 2009). Together with two sister species, Glycyrrhiza glabra L. and Glycyrrhiza inflata Batalin, G. uralensis is one of important traditional Chinese medicine used as sweetener (Rizzato et al., 2017), and has long been cultivated in China and Korea (Hayashi and Sudo, 2009; Park et al., 2020a). Because G. uralensis is recognized as the best species among three Glycyrrhiza species with recent decease of production of $G$. uralenesis in inner Mongolia, the closely related species of $G$. uralensis have been considered as alternative species (Lee et al., 2010; Kim et al., 2019a). Due to this importance, multiple number of chloroplast genomes have been sequenced till now (Kang et al., 2018; Jia et al., 2019) and several molecular markers, such as simple sequence repeats, have been developed (Hayashi et al., 2005; Lee et al., 2019; Hantemirova et al., 2020). Moreover, various useful compounds, including antibacterial and antithrombotic compounds, have been identified from G. uralensis (He et al., 2006; Kwon et al., 2010; Lee et al., 2010; Tao et al., 2012; Adianti et al., 2014). In addition, natural hybrids of Glycyrrhiza were reported (Chen et al., 2020) and some cultivars through hybridization were developed (Lee et al., 2017). Here, we completed the chloroplast genome of $G$. uralensis cultivated in Korea to investigate intraspecific variations on chloroplast genomes and its intraspecific phylogeny.

\section{Materials and Methods}

\section{Plant material}

We collected the $G$. uralensis in Godo 9-gil, Iksan-si, Jeollabuk-do, in Korea $\left(36^{\circ} 00^{\prime} 14.45^{\prime \prime} \mathrm{N}, 127^{\circ} 03^{\prime} 49.47^{\prime \prime E}\right)$. A voucher and isolated DNA was deposited in the InfoBoss Cyber Herbarium (IN, the voucher number IB-01093).

\footnotetext{
${ }^{* *}$ These authors contributed equally to this work.

*Author for correspondence: starflr@infoboss.co.kr (J. Park), gwlee@jami.re.kr (G. W. Lee)

Open Access http://e-kjpt.org, (C) 2021 the Korean Society of Plant Taxonomists. This is an open-access article distributed under the terms of the Creative Commons Attribution Non-Commercial License (http://creativecommons.org/licenses/by-ne/4.0) which permits unrestricted non-commercial use, distribution, and reproduction in any medium, provided the original work is properly cited.
} 


\section{DNA extraction and chloroplast genome determination}

Total DNA was extracted from fresh root by using a DNeasy Plant Mini Kit (QIAGEN, Hilden, Germany). Genome sequencing was performed using NovaSeq6000 at Macrogen Inc., Korea, resulting in $15.8 \mathrm{Gbp}$ raw reads. De novo assembly was done by Velvet v1.2.10 (Zerbino and Birney, 2008) and GapCloser v1.12 (Zhao et al., 2011). Assembled sequences were modified and confirmed by BWA v0.7.17 (Li, 2013) and SAMtools v1.9 (Li et al., 2009). All analyses were conducted in the Genome Information System (GeIS; http://geis.infoboss.co.kr/) used in the previous studies (Bum et al., 2020; Kim et al., 2021).

Genome annotation was conducted based on another chloroplast genome of G. uralensis (MT120790) with Geneious Prime 2020.2.4 (Biomatters Ltd., Auckland, New Zealand). A circular map of G. uralensis chloroplast genome was drawn using OGDRAW v1.31 (Greiner et al., 2019).

\section{Identification of intraspecific variations}

Single nucleotide polymorphisms (SNPs) and insertions and deletions (INDELs) were identified from the multiple sequence alignment of the six G. uralensis chloroplast genomes conducted by MAFFT 7.450 (Katoh and Standley, 2013) with 'Find variations/SNPs' implemented in Geneious Prime 2020.2.4 (Biomatters Ltd.). In addition, multiple sequence alignments from eleven Glycyrrhiza species were used in the same way (Table 1). Moreover, pair-wise alignments of the selected chloroplast genomes (Table 1) were also utilized in the same way. This method has been used in the previous studies investigating intraspecific variations on organelle genomes
(Choi et al., 2021; Park et al., 2021c, 2021d). INDEL region was defined as the continuous INDELs.

\section{Nucleotide diversity analysis of chloroplast genomes}

We calculated nucleotide diversity in the six chloroplast genomes of $G$. uralensis using the method proposed by Nei and Li (1979) with the Perl script. Nucleotide diversities were scanned along the genome with 500-bp window size and 200bp step size for overlapped sliding windows. Our script for nucleotide diversity calculation has been utilized in the previous studies (Kim et al., 2019b; Lee et al., 2020a, 2020b, 2020c; Park et al., 2021e; Park and Xi, 2021).

\section{Phylogenetic analysis}

Maximum-likelihood (ML) and Bayesian inference (BI) phylogenetic trees were constructed based on the multiple sequence alignment of 57 Glycyrrhiza chloroplast genomes by MAFFT v7.450 (Katoh and Standley, 2013). The ML tree was reconstructed in MEGA X with 1,000 bootstrap repeats (Kumar et al., 2018). In the ML analysis, a heuristic search was used with nearest-neighbor interchange branch swapping, TVM + $\mathrm{F}+\mathrm{R} 4$ model, and uniform rates among sites. All other options used the default settings. The posterior probability of each node was estimated by BI using MrBayes v3.2.6 (Huelsenbeck and Ronquist, 2001) plug-in implemented in Geneious Prime 2020.2.4 (Biomatters Ltd.). The HKY85 model with gamma rates was used as a molecular model. A Markov chain Monte Carlo algorithm was employed for 10,000,000 generations, sampling trees every 200 generations, with four chains running

Table 1. List of number of intraspecific variations identified from chloroplast genomes of the twelve Glycyrrhiza species including G. uralenesis.

\begin{tabular}{lcccc}
\hline \hline \multicolumn{1}{c}{ Species } & No. of SNPs & No. of INDEL regions & INDEL coverage (bp) & Alignment length (bp) \\
\hline G. uralensis Fisch. & 150 & 10 & 75 & 126,304 \\
G. aspera Pall. & 43 & 22 & 79 & 127,890 \\
G. astragalina Gillies ex Hook.\& Arn. & 95 & 44 & 475 & 127,201 \\
G. echinata L. & 11 & 8 & 15 & 128,072 \\
G. foetida Desf. & 13 & 25 & 166 & 127,857 \\
G. glabra L. & 102 & 61 & 623 & 128,132 \\
G. glandulifera Waldst. \& Kit. & 29 & 24 & 77 & 128,000 \\
G. inflata Batalin. & 25 & 35 & 162 & 127,960 \\
G. lepidota (Nutt.) Pursh & 1163 & 281 & 2339 & 128,805 \\
G. pallidiflora Maxim. & 49 & 39 & 676 & 127,790 \\
G. squamulosa Franch. & 48 & 20 & 125 & 127,788 \\
G. triphylla Fisch. \& CAMey. & 100 & 38 & 205 & 127,856 \\
\hline
\end{tabular}


simultaneously. Trees from the first 2,500,000 generations were discarded as burn-in.

\section{Results and Discussion}

The chloroplast genome of G. uralensis (GenBank accession: MZ066516) isolated in Korea is 127,689 bp long, which is shorter than those of typical plant chloroplast genomes. This is caused by lack of one of inverted repeat regions in the typical plant chloroplast genomes (Fig. 1), which is a common phenomenon in genus Glycyrrhiza (Kang et al., 2018). However, Abrus chloroplast (GenBank accession: MT328396), neighbor genus of Glycyrrhiza (Hayashi et al., 1998), presents a typical structure of chloroplast genome, indicating that this atypical structure is specific to Glycyrrhiza. It contains 110 genes consisting of 76 protein-coding genes (PCGs), four ribosomal RNAs (rRNAs), and 30 transfer RNAs (tRNAs) (Fig. 1).

A total of 150 SNPs and 10 INDEL regions covering $75 \mathrm{bp}$ were identified from the six G. uralensis chloroplast genomes. Eighteen out of 76 PCGs, including $\operatorname{mat} K, a t p B, n d h K, p s a B$,

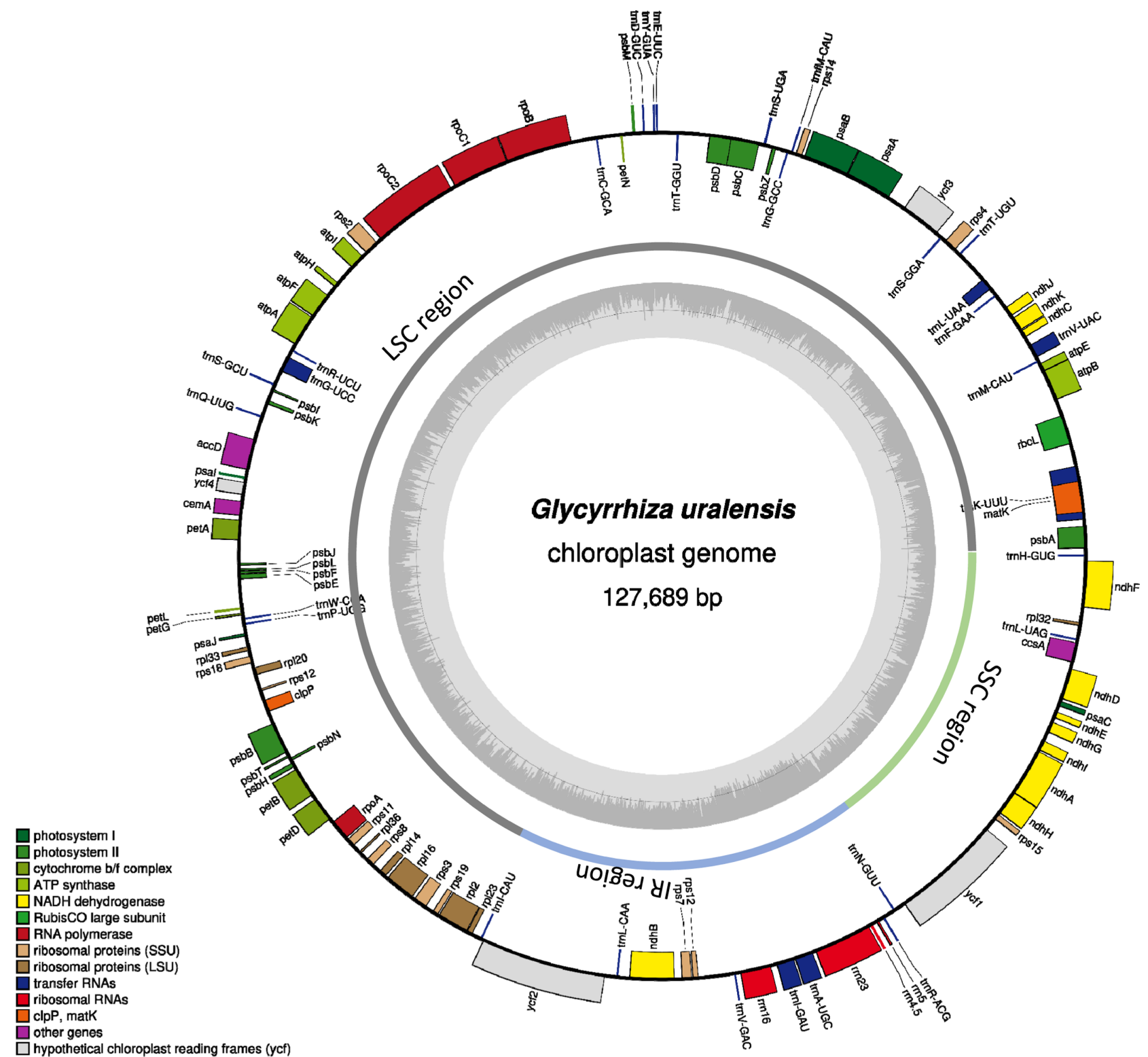

Fig. 1. Circular map of chloroplast genome of Glycyrrhiza uralensis isolated in Korea. Gene shown outside are transcribed clockwise, and inside the circle are transcribed counter clockwise. Genes are color-coded to distinguish different functional groups. The dark grey and the light grey plot in the inner circle correspond to the GC content and AT content, respectively. Inside chloroplast genome, the three arcs of which colors are grey, light green, and light blue indicate large single copy (LSC), small single copy (SSC), and inverted repeat (IR) regions. 
psbC, rpoB, rpoC1, rpoC2, atpF, atpA, rpl20, psbB, rps3, ycf2, $y c f 1, n d h L, \operatorname{css} A$, and $n d h F$, cover at least one SNPs. The number of PCGs containing at least one SNP is slightly larger than that of Chenopodium album L. (Park et al., 2021b); however, the ratio of non-synonymous to synonymous SNPs is $15: 19$, which is typical in anigosperms. The genes containing non-synonymous SNPs, matK, atpB, ndhK, rpoCl, rps3, ycfl, and $\operatorname{css} A$, can be a candidate to develop Glycyrrhiza-specific molecular marker to evaluate their generic background.

To evaluate the numbers of SNPs and INDELs identified from G. uralensis chloroplast genomes, we investigated intraspecific variations of eleven Glycyrrhiza species (Table 1) using the

INDEL coverage (bp)

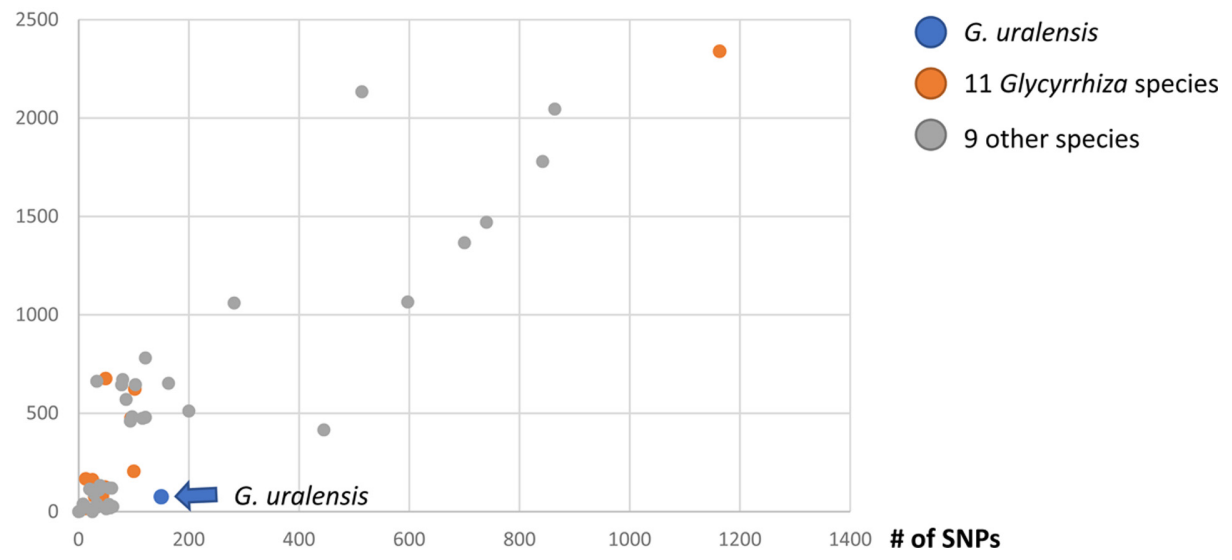

Fig. 2. Scattered plot of number of single nucleotide polymorphisms (SNPs) and insertion and deletion (INDEL) coverage (bp) identified from the twelve Glycyrrhiza species and nine species. X-axis is number of SNPs and Y-axis is INDEL coverage. Blue dot indicates the number of SNPs and INDEL coverage identified from G. uralensis, orange dots were from eleven Glycyrrhiza species, and grey dots were from those of nine plant species identified based on pair-wise alignment of complete chloroplast genomes.

Table 2. List of number of intraspecific variations identified from chloroplast genomes of the various plant species.

\begin{tabular}{ccccccc}
\hline Family & Species & $\begin{array}{c}\text { No. of } \\
\text { SNPs }\end{array}$ & $\begin{array}{c}\text { No. of INDEL } \\
\text { regions }\end{array}$ & $\begin{array}{c}\text { INDEL } \\
\text { coverage (bp) }\end{array}$ & $\begin{array}{c}\text { Alignment length } \\
\text { (bp) }\end{array}$ & Reference \\
\hline Asteraceae & Chrysanthemum zawadskii Herbich. & 182 & 71 & 426 & 151,359 & $\begin{array}{c}\text { Baek et al. (2021), } \\
\text { Hongmei et al. } \\
\text { (2021) }\end{array}$ \\
& & 108 & 45 & 251 & 151,221 & Park et al. (2020b) \\
Brasicaceae & Arabidopsis thaliana L. & 55 & 10 & 36 & 154,485 & 154,938 \\
& & 116 & 6 & 474 & 154,492 \\
& 10 & 12 & 33 & 154,478 \\
& 50 & 4 & 14 & 154,550 \\
& 56 & 44 & 120 & 154,945 \\
& 97 & 8 & 482 & 154,491 \\
& 62 & 7 & 25 & 154,485 \\
& 33 & 6 & 22 & 154,559 \\
& 39 & 49 & 132 & 154,952 \\
& 121 & 10 & 479 & 154,938 \\
& 94 & 2 & 460 & 155,012 \\
& 86 & 46 & 570 & 154,492 \\
& 57 & 8 & 19 & 154,561 \\
& 60 & 49 & 44 & 119 & 154,552 \\
& 37 & & &
\end{tabular}


Table 2. Continued.

\begin{tabular}{|c|c|c|c|c|c|c|}
\hline Family & Species & $\begin{array}{l}\text { No. of } \\
\text { SNPs }\end{array}$ & $\begin{array}{l}\text { No. of INDEL } \\
\text { regions }\end{array}$ & $\begin{array}{c}\text { INDEL } \\
\text { coverage (bp) }\end{array}$ & $\begin{array}{l}\text { Alignment length } \\
\text { (bp) }\end{array}$ & Reference \\
\hline \multirow[t]{10}{*}{ Orchidaceae } & \multirow[t]{10}{*}{ Goodyera schlechtendaliana Rchb. F. } & 200 & 59 & 511 & 154,607 & \multirow{10}{*}{$\begin{array}{l}\text { Oh et al. (2019a, } \\
\text { 2019b) }\end{array}$} \\
\hline & & 842 & 130 & 1,779 & 154,964 & \\
\hline & & 740 & 95 & 1,470 & 154,820 & \\
\hline & & 514 & 110 & 2,133 & 155,322 & \\
\hline & & 700 & 112 & 1,366 & 154,665 & \\
\hline & & 597 & 72 & 1,065 & 154,525 & \\
\hline & & 445 & 29 & 415 & 154,119 & \\
\hline & & 864 & 123 & 2,045 & 155,027 & \\
\hline & & 282 & 86 & 1,060 & 154,261 & \\
\hline & & 163 & 43 & 652 & 154,067 & \\
\hline Rosaceae & Pyrus ussuriensis Maxim. & 121 & 61 & 781 & 160,727 & Cho et al. (2019) \\
\hline \multirow[t]{3}{*}{ Fagaceae } & \multirow[t]{3}{*}{ Fagus multinervis Nakai } & 2 & 2 & 2 & 158,350 & \multirow{3}{*}{$\begin{array}{l}\text { Park and Oh (2020) } \\
\text { Yang et al. (2020) }\end{array}$} \\
\hline & & 2 & 3 & 3 & 158,350 & \\
\hline & & 0 & 0 & 0 & 158,348 & \\
\hline Schisandraceae & Illicium anisatum $\mathrm{L}$. & 20 & 17 & 114 & 146,939 & Park et al. (2019a) \\
\hline \multirow[t]{10}{*}{ Theaceae } & \multirow[t]{10}{*}{ Camellia japonica $\mathrm{L}$. } & 25 & 2 & 2 & 156,976 & \multirow{10}{*}{$\begin{array}{c}\text { Park et al. (2019c, } \\
\text { 2019d) }\end{array}$} \\
\hline & & 25 & 2 & 2 & 156,972 & \\
\hline & & 25 & 0 & 0 & 156,971 & \\
\hline & & 8 & 7 & 36 & 157,004 & \\
\hline & & 8 & 9 & 38 & 157,005 & \\
\hline & & 33 & 9 & 38 & 157,005 & \\
\hline & & 78 & 60 & 644 & 157,110 & \\
\hline & & 78 & 61 & 645 & 157,111 & \\
\hline & & 103 & 61 & 645 & 157,111 & \\
\hline & & 80 & 61 & 671 & 157,139 & \\
\hline Campanulaceae & Campanula takesimana Nakai & 33 & 39 & 662 & 169,737 & Park et al. (2021a) \\
\hline Poaceae & Zoysia matrella (L.) Merr. & 28 & 56 & 91 & 135,894 & $\begin{array}{l}\text { Lee and Park } \\
\text { (2021) }\end{array}$ \\
\hline
\end{tabular}

available complete chloroplast genomes. The numbers of SNPs ranged from 11 to 1,163 and those of INDEL regions were from 8 to 281 (15 to 2,339 bp in length) (Fig. 2). Many of numbers of intraspecific variations of the other plant species showed similar or smaller than those of G. uralensis except Goodyera schlechtendaliana Rchb. F. (Fig. 2, Table 2). It indicates that the number of SNPs of G. uralensis is in the middle, but INDEL coverage is relatively small. However, genetic diversity of chloroplast genomes should be investigated further based on well-designed sampling strategy considering with the number of samples and their locations together.

Nucleotide diversity of six G. uralensis chloroplast genomes was calculated to investigated hotspot of nucleotide diversity. Average nucleotide diversity was 0.0002216 (Fig. 3A) which is higher than those of $A$. thaliana (0.000017) isolated from several countries (Park et al., 2020b) and C. album (0.0000625) isolated in the Korean Peninsula (Park et al., 2021b). This high intraspecific nucleotide diveristy may be caused by different genetic backgrounds of $G$. uralensis, in which accessions are separated in two indepdendent clades in the phylogenetic tree (Fig. 3A). Interestingly 5 ' region of $a t p B$ contained three nonsynonymous SNPs (Fig. 3B), which is congruent to the studies presenting that $a t p B$ is one of positive selected genes (Xie et al., 2018; Lee and Park, 2021; Zhang et al., 2021). In addition, 


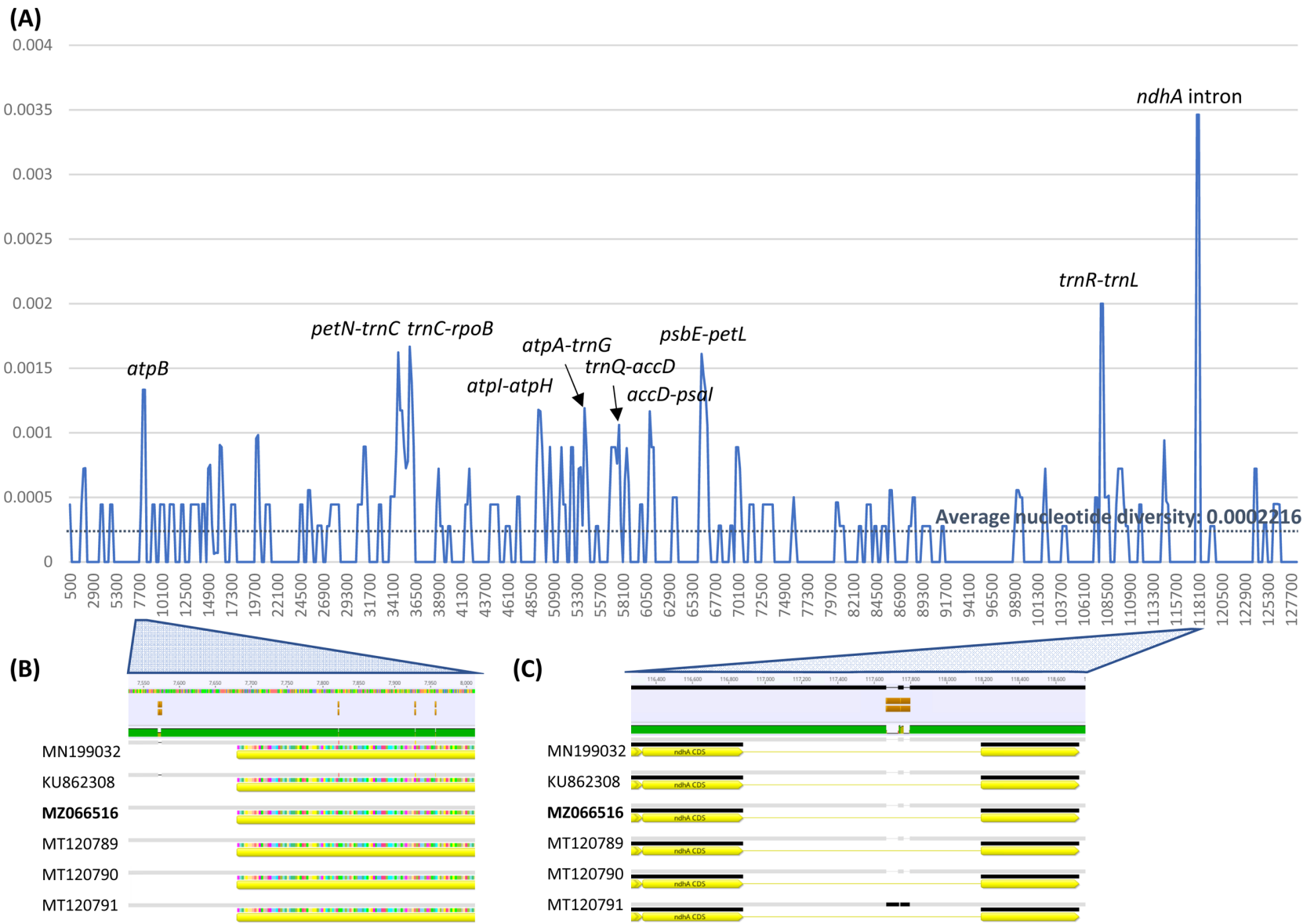

Fig. 3. Nucleotide diversity calculated from the six G. uralensis chloroplast genomes. A. X-axis indicates coordination of chloroplast genome and Y-axis indicates nucleotide diversity of each window. Average nucleotide diversity was displayed as dark blue color and labels on graph indicate nearby gene names of the peak of which nucleotide diversity is above 0.001 . B. Enlarged diagram shows multiple sequence alignment in 5 ' region of $a t p B$. C. Enlarged diagram shows multiple sequence alignment in 5 ' region of $n d h A$ intron.

intron region of $n d h A$ also displayed high nucleotide diversity, caused by one big insertion region from MT120791 (Fig. 3C). This phenomenon was also observed in various chloroplast genomes, such as A. thaliana (Park et al., 2020b), Viburnum amplificatum Kern (Park et al., 2020c), and Coffea arabica L. (Park et al., 2019b). Furthermore, intergenic regions of petNtrnC, trnC-rpoB, atpI-atpH, atpA-trnG, trnQ-accD, accD-psaI, psbE-petL, and trnR-trnL presented high nucleotide diversity (Fig. 3A), which can be used for developing molecular markers to distinguish G. uralensis populations or cultivars.

Fifty-seven Glycyrrhiza chloroplast genomes with one outgroup species of Abrus pulchellus subsp. cantoniensis (Hance) Verdc., were used for constructing phylogenetic trees. Both phylogenetic trees display that six $G$. uralensis are clustered into the two clades including one G. glabra (GenBank accession: MG736059) with high supportive values of ML and BI trees except one low bootstrap value of ML (Fig. 4). Phylogenetic position of $G$. uralenesis is congruent to the previous phylogenetic studies (Hayashi et al., 1998, 2000; Liu et al., 2019; Duan et al., 2020). In addition, one G. glabra chloroplast (GenBank accession: MG736059) was found in the clade of G. uralensis (Fig. 4). One of possibility of this chloroplast can be misidentification because G. glabra was in the same clade with G. inflata in the previous studies (Hayashi et al., 1998, 2000; Liu et al., 2019; Duan et al., 2020). Moreover, two G. uralensis chloroplast genomes (GenBank accessions: KU862308 and MN199032) isolated in Korea and China (Jia et al., 2019), respectively, were clustered separately (Fig. 4), suggesting that geographical origin of G. uralensis might not be related to these two clades due to its cultivation in East-Asian countries (Yamamoto et al., 2003; Hayashi et al., 2005). Interestingly, non-synonymous SNPs identified only in the two G. uralensis chloroplast genomes (GenBank accessions: KU862308 and MN199032) were found in $a t p B$ (Fig. 3B) as well as $n d h K$, $r p o C 2$, and $y c f 1$. In addition, synonymous SNPs from those chloroplast genomes were also identified in $p s b$, $p s b$, rpoB, 


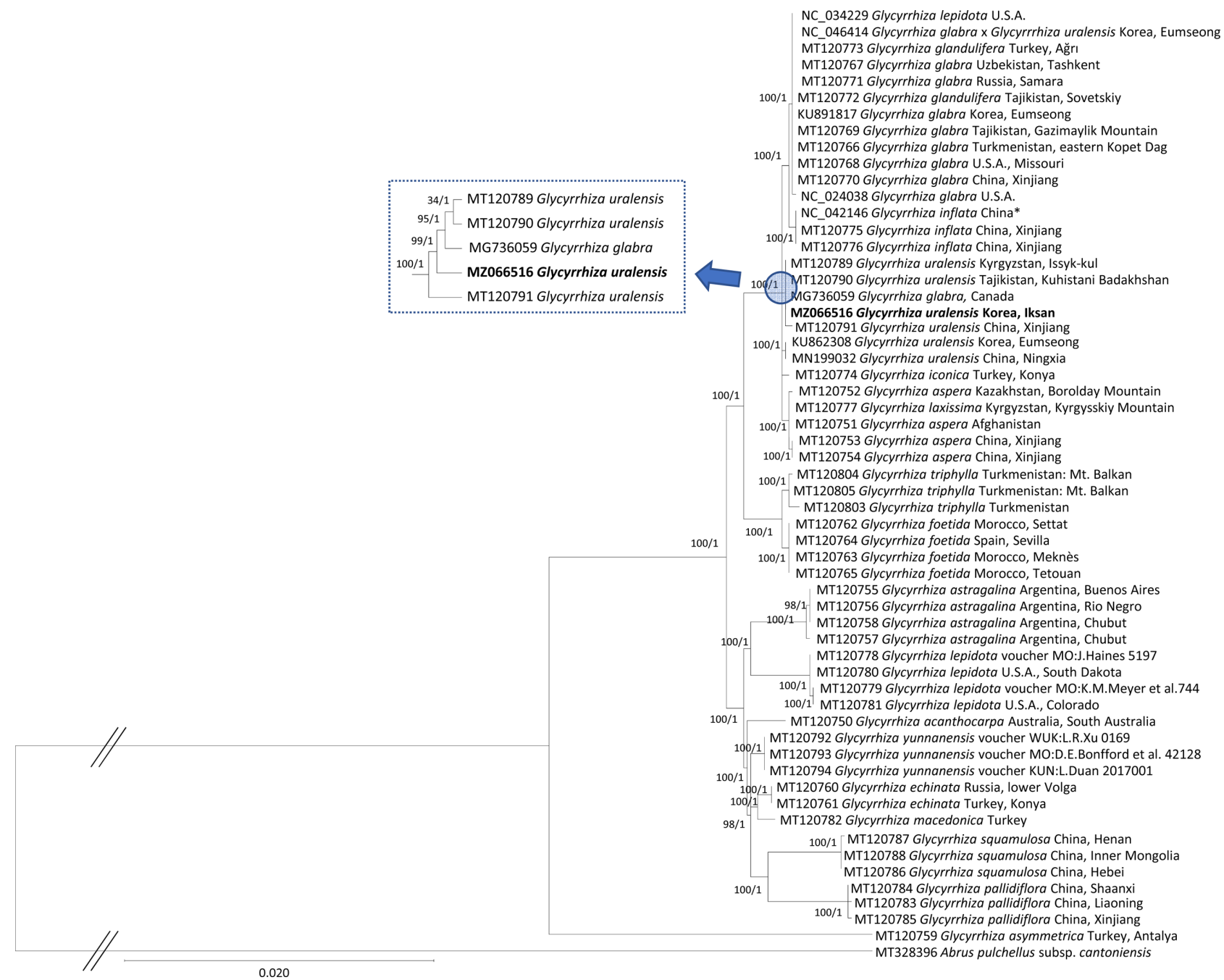

Fig. 4. Maximum-likelihood (ML) and Bayesian inference (BI) phylogenetic trees based on the complete chloroplast genomes of 57 Glycyrrhiza and one outgroup taxon, Abrus pulchellus subsp. cantoniensis. Phylogenetic tree was drawn based on ML tree. The numbers above branches indicate bootstrap values of ML tree and posterior probabilities of BI tree, respectively. Locations of each chloroplast genome were displayed after species name, originated from the published papers (Sabir et al., 2014; Kang et al., 2018; Bang et al., 2019; Jia et al., 2019; Duan et al., 2020) or source section of GenBank files of unpublished chloroplast genomes. Star mark in the NC_042146 indicates that location is not available and it is from the location of institute. The clade containing MZ066516 chloroplast was enlarged to make it easy to show the topology.

rpoC1, rpoC2, atp $A, a c c D, p s b B$, and $n d h F$, which may contribute to forming the two separate clades. G. uralensis chloroplast genome assembled in this study provides insights of its intraspecific features, which will be helpful for understanding the structure of cultivated and native G. uralensis populations in detail.

ORCID: Mi-Hee KIM http://orcid.org/0000-0001-5846-1887; Suhyeon PARK http://orcid.org/0000-0002-3321-1950; Junho LEE http://orcid.org/0000-0002-4443-3401; Jinwook BAEK http://orcid.org/0000-0001-5027-0912; Jongsun PARK http:// orcid.org/0000-0003-0786-4701; Gun Woong LEE https:// orcid.org/0000-0001-7091-8106

\section{Acknowledgments}

This study was carried out with the support of the Ministry of Small and Medium-sized Enterprises (SMEs) and Startups (MSS), Korea, under the "Regional Specialized Industry Development Plus Program (R\&D, S2913418)" supervised by the Korea Institute for Advancement of Technology (KIAT).

\section{Conflicts of Interest}

The authors declare that there are no conflicts of interest. 


\section{Literature Cited}

Adianti, M., C. Aoki, M. Komoto, L. Deng, I. Shoji, T. S. Wahyuni, M. I. Lusida, Soetjipto, H. Fuchino, N. Kawahara and H. Hotta. 2014. Anti-hepatitis C virus compounds obtained from Glycyrrhiza uralensis and other Glycyrrhiza species. Microbiology and Immunology 58: 180-187.

Baek J., S. Park, J. Lee, J. Min, J. Park and G. W. Lee. 2021. The complete chloroplast genome of Chrysanthemum zawadskii Herbich (Asteraceae) isolated in Korea. Mitochondrial DNA Part B Resources 6: 1956-1958.

Bang, J. H., C. E. Hong, S. Raveendar, K. H. Bang, K. H. Ma, S. W. Kwon, H. Ryu, I. H. Jo and J.-W. Chung. 2019. Development of genomic simple sequence repeat markers for Glycyrrhiza lepidota and cross-amplification of other Glycyrrhiza species. PeerJ 7: e7479.

Bum, H.-M., H. K. Kim, N. S. Kim, W. Kwon and J. Park. 2020. The complete chloroplast genome of Douinia plicata (Lindb.) Konstant. \& Vilnet (Scapaniaceae, Jungermanniales). Mitochondrial DNA Part B Resources 5: 3698-3700.

Chen, W.-Q., J.-H. Lu, Q.-Q. Wang, Q. Xin and Y. Xu. 2020. Numerical taxonomic study of morphological characteristics of species in Glycyrrhiza hybrid zones. Acta Prataculturae Sinica 29: 14-26.

Cho, M.-S., Y. Kim, S.-C. Kim and J. Park. 2019. The complete chloroplast genome of Korean Pyrus ussuriensis Maxim. (Rosaceae): Providing genetic background of two types of $P$. ussuriensis. Mitochondrial DNA Part B Resources 4: 2424 2425.

Choi, N. J., H. Xi and J. Park. 2021. A comparative analyses of the complete mitochondrial genomes of fungal endosymbionts in Sogatella furcifera, white-backed planthoppers. International Journal of Genomics 2021: 6652508.

Duan, L., A. J. Harris, C. Su, Z.-R. Zhang, E. Arslan, K. Ertuğrul, P. K. Loc, H. Hayashi, J. Wen and H.-F. Chen. 2020. Chloroplast phylogenomics reveals the intercontinental biogeographic history of the Liquorice genus (Leguminosae: Glycyrrhiza). Frontiers in Plant Science 11: 793.

Greiner, S., P. Lehwark and R. Bock. 2019. OrganellarGenomeDRAW (OGDRAW) version 1.3.1: Expanded toolkit for the graphical visualization of organellar genomes. Nucleic Acids Research 47: W59-W64.

Hantemirova, E., A. Y. Belyaev, O. S. Korchagina and I. A. Laletina. 2020. Genetic differentiation and phylogenetic relationships of Glycyrrhiza glabra L., G. uralensis Fisch., and G. korshinskyi Grig. inferred from chloroplast DNA variation. Russian Journal of Genetics 56: 810-821.

Hayashi, H., N. Hosono, M. Kondo, N. Hiraoka and Y. Ikeshiro.
1998. Phylogenetic relationship of Glycyrrhiza plants based on rbcL sequences. Biological and Pharmaceutical Bulletin 21: 782-783.

Hayashi, H., N. Hosono, M. Kondo, N. Hiroka, Y. Ikeshiro, M. Shibano, G. Kusano, H. Yamamoto, T. Tanaka and K. Inoue. 2000. Phylogenetic relationship of six Glycyrrhiza species based on rbcL sequences and chemical constituents. Biological and Pharmaceutical Bulletin 23: 602-606.

Hayashi, H., K. Inoue, K. Ozaki and H. Watanabe. 2005. Comparative analysis of ten strains of Glycyrrhiza uralensis cultivated in Japan. Biological and Pharmaceutical Bulletin 28: 1113-1116.

Hayashi, H. and H. Sudo. 2009. Economic importance of licorice. Plant Biotechnology 26: 101-104.

He, J., L. Chen, D. Heber, W. Shi and Q.-Y. Lu. 2006. Antibacterial compounds from Glycyrrhiza uralensis. Journal of Natural Products 69: 121-124.

Hongmei, S., H. Wenrui, H. Dianyun, X. Yang. 2021. Complete chloroplast genome sequence of Dendranthema zawadskii Herbich. Mitochondrial DNA Part B Resources 6: 2117-2119.

Huelsenbeck, J. P. and F. Ronquist. 2001. MRBAYES: Bayesian inference of phylogenetic trees. Bioinformatics 17: 754-755.

Jia, G., P. Li, Q. Zhu and L. Peng. 2019. Characterization of the complete chloroplast genome of Glycyrrhiza uralensis (Leguminosae), a traditional Chinese medicine. Mitochondrial DNA Part B Resources 4: 3040-3041.

Kang, S.-H., J.-H. Lee, H.-O. Lee, B. O. Ahn, S. Y. Won, S.-H. Sohn and J. S. Kim. 2018. Complete chloroplast genome and 45S nrDNA sequences of the medicinal plant species Glycyrrhiza glabra and Glycyrrhiza uralensis. Genes and Genetic Systems 93: 83-89.

Katoh, K. and D. M. Standley. 2013. MAFFT multiple sequence alignment software version 7: Improvements in performance and usability. Molecular Biology and Evolution 30: 772-780.

Kim, M., H. Xi and J. Park. 2021. Genome-wide comparative analyses of GATA transcription factors among 19 Arabidopsis ecotype genomes: Intraspecific characteristics of GATA transcription factors. PLoS ONE 16: e0252181.

Kim, Y.-S., C.-G. Park, G. Choi, J.-K. Chang, J.-H. Lee and Y.-S. Ju. 2019a. Comparative study of external-intenal morphological shape in origins and hybrids for Glycyrrhizae Radix et Rhizoma. The Korea Journal of Herbology 34: 1-12.

Kim, Y., J. Park and Y. Chung. 2019b. Comparative analysis of chloroplast genome of Dysphania ambrosioides (L.) Mosyakin \& Clemants understanding phylogenetic relationship in genus Dysphania R. Br. Korean Journal of Plant Resources 32: 644-668.

Kumar, S., G. Stecher, M. Li, C. Knyaz and K. Tamura. 2018. 
MEGA X: Molecular Evolutionary Genetics Analysis across computing platforms. Molecular Biology and Evolution 35: $1547-1549$.

Kwon, H.-J., H.-H. Kim, Y. B. Ryu, J. H. Kim, H. J. Jeong, S.-W. Lee, J. S. Chang, K.-O. Cho, M.-C. Rho, S.-J. Park and W. S. Lee. 2010. In vitro anti-rotavirus activity of polyphenol compounds isolated from the roots of Glycyrrhiza uralensis. Bioorganic and Medicinal Chemistry 18: 7668-7674.

Lee, B. and J. Park. 2021. The complete chloroplast genome of Zoysia matrella (L.) Merr. isolated in Korea (Poaceae): Investigation of intraspecific variations on chloroplast genomes. Mitochondrial DNA Part B Resources 6: 572-574.

Lee, H., J. Park, H. Xi, G.-S. Lee, I. Kim, J. Park and W. Lee. 2020a. The complete mitochondrial genome of Ricania speculum (Walker, 1851) (Hemiptera: Ricaniidae): Investigation of intraspecific variations on mitochondrial genome. Mitochondrial DNA Part B Resources 5: 3796-3798.

Lee, J., J. Park, H. Xi and J. Park. 2020b. Comprehensive analyses of the complete mitochondrial genome of Figulus binodulus (Coleoptera: Lucanidae). Journal of Insect Science 20: 10.

Lee, J.-H., M.-W. Oh, S.-H. Lee, C.-G. Park, J.-T. Jeong, J.-W. Han, K.-H. Ma and J.-K. Chang. 2020c. 'Wongam', a licorice interspecific hybrid cultivar with high yield. Korean Society of Breeding Science 52: 454-459.

Lee, K. J., S. Raveendar, J. S. Choi, J. Gil, J. H. Lee, Y.-S. So and J.-W. Chung. 2019. Development of chloroplast microsatellite markers for identification of Glycyrrhiza species. Plant Genetic Resources 17: 95-98.

Lee, S.-H., Y. B. Kim, J.-M. Lee, J.-H. Lee, C.-B. Park, J.-W. Bang, H.-W. Choi, Y. Hur and C.-G. Park. 2017. Cultivation and characteristics of licorice F1 (Glycyrrhiza glabra $\times$ G. uralensis) lines. Horticultural Science and Technology 35: 276286.

Lee, Y. S., S. H. Kim, S. H. Jung, J. K. Kim, C.-H. Pan and S. S. Lim. 2010. Aldose reductase inhibitory compounds from Glycyrrhiza uralensis. Biological and Pharmaceutical Bulletin 33: 917-921.

Li, H. 2013. Aligning sequence reads, clone sequences and assembly contigs with BWA-MEM. arXiv preprint at: https:// arxiv.org/abs/1303.3997.

Li, H., B. Handsaker, A. Wysoker, T. Fennell, J. Ruan, N. Homer, G. Marth, G. Abecasis, R. Durbin and 1000 Genome Project Data Processing Subgroup. 2009. The sequence alignment/ map format and SAMtools. Bioinformatics 25: 2078-2079.

Liu, Y., Y. Geng, M. Song, P. Zhang, J. Hou and W. Wang. 2019. Genetic structure and diversity of Glycyrrhiza populations based on transcriptome SSR markers. Plant Molecular Biology Reporter 37: 401-412.
Nei, M. and W. H. Li. 1979. Mathematical model for studying genetic variation in terms of restriction endonucleases. Proceedings of the National Academy of Sciences of the United States of America 76: 5269-5273.

Oh, S.-H., H. J. Suh, J. Park, Y. Kim and S. Kim. 2019a. The complete chloroplast genome sequence of a morphotype of Goodyera schlechtendaliana (Orchidaceae) with the column appendages. Mitochondrial DNA Part B 4: 626-627.

Oh, S.-H., H. J. Suh, J. Park, Y. Kim and S. Kim. 2019b. The complete chloroplast genome sequence of Goodyera schlechtendaliana in Korea (Orchidaceae). Mitochondrial DNA Part B Resources 4: 2692-2693.

Park, J., Y. Kim and H. Xi. 2019a. The complete chloroplast genome of aniseed tree, Illicium anisatum L. (Schisandraceae). Mitochondrial DNA Part B Resources 4: 1023-1024.

Park, J., Y. Kim, H. Xi and K.-I. Heo. 2019b. The complete chloroplast genome of ornamental coffee tree, Coffea arabica L. (Rubiaceae). Mitochondrial DNA Part B Resources 4: 10591060.

Park, J., Y. Kim, H. Xi, Y. J. Oh, K. M. Hamn and J. Ko. 2019c. The complete chloroplast genome of common camellia tree, Camellia japonica L. (Theaceae), adapted to cold environment in Korea. Mitochondrial DNA Part B Resources 4: $1038-1040$.

Park, J., Y. Kim, H. Xi, Y. J. Oh, K. M. Hamn and J. Ko. 2019d. The complete chloroplast genome of common camellia tree in Jeju island, Korea, Camellia japonica L. (Theaceae): Intraspecies variations on common camellia chloroplast genomes. Mitochondrial DNA Part B Resources 4: 1292-1293.

Park, J., J.-H. An, Y. Kim, D. Kim, B.-G. Yang and T. Kim. 2020a. Database of National Species List of Korea: The taxonomical systematics platform for managing scientific names of Korean native species. Journal of Species Research 9: 233-246.

Park, J. and S.-H. Oh. 2020. A second complete chloroplast genome sequenceof Fagus multinervis Nakai (Fagaceae): Intraspecific variations on chloroplast genome. Mitochondrial DNA Part B Resources 5: 1868-1869.

Park, J. and H. Xi. 2021. Investigation of nucleotide diversity based on 17 sea cucumber mitochondrial genomes and assessment of sea cucumber mitochondrial gene markers. Advances in Oceanography and Marine Biology 2: 2021.

Park, J., H. Xi and Y. Kim. 2020b. The complete chloroplast genome of Arabidopsis thaliana isolated in Korea (Brassicaceae): An investigation of intraspecific variations of the chloroplast genome of Korean A. thaliana. International Journal of Genomics 2020: 3236461.

Park, J., H. Xi and S.-H. Oh. 2020c. Comparative chloroplast genomics and phylogenetic analysis of the Viburnum dila- 
tatum complex (Adoxaceae) in Korea. Korean Journal of Plant Taxonomy 50: 8-16.

Park, J., Y. Bae, B.-Y. Kim, G.-H. Nam, J.-M. Park, B.-Y. Lee, H.J. Suh and S.-H. Oh. 2021a. The complete chloroplast genome of Campanula takesimana Nakai from Dokdo Island in Korea (Campanulaceae). Mitochondrial DNA Part B Resources 6: 135-137.

Park, J., J. Min, Y. Kim and Y. Chung. 2021b. The comparative analyses of six complete chloroplast genomes of morphologically diverse Chenopodium album L. (Amaranthaceae) collected in Korea. International Journal of Genomics 2021: 6643444.

Park, J., Y. Kim, W. Kwon, H, Xi and C.-H. Park. 2021c. The complete chloroplast genome sequence of new species candidate of Plantago depressa Willd. in Korea (Plantaginaceae). Mitochondrial DNA Part B Resources 6: 1961-1963.

Park, J., H. Xi and Y. Kim. 2021d. The complete mitochondrial genome of Arabidopsis thaliana (Brassicaceae) isolated in Korea. Korean Journal of Plant Taxonomy 51: 176-180.

Park, J., H. Xi, J. Park and B. Y. Seo. 2021e. a new mitochondrial genome of Sogatella furcifera (Horváth) (Hemiptera: Delphacidae) and mitogenome-wide investigation on polymorphisms. Insects 12: 1066.

Rizzato, G., E. Scalabrin, M. Radaelli, G. Capodaglio and O. Piccolo. 2017. A new exploration of licorice metabolome. Food Chemistry 221: 959-968.

Sabir, J., E. Schwarz, N. Ellison, J. Zhang, N. A. Baeshen, M. Mutwakil, R. Jansen and T. Ruhlman. 2014. Evolutionary and biotechnology implications of plastid genome variation in the inverted-repeat-lacking clade of legumes. Plant Biotechnology Journal 12: 743-754.

Tao, W.-W., J.-A. Duan, N.-Y. Yang, Y.-P. Tang, M.-Z. Liu and Y.F. Qian. 2012. Antithrombotic phenolic compounds from Glycyrrhiza uralensis. Fitoterapia 83: 422-425.

Xie, D.-F., Y. Yu, Y.-Q. Deng, J. Li, H.-Y. Liu, S.-D. Zhou and X.J. He. 2018. Comparative analysis of the chloroplast genomes of the Chinese endemic genus Urophysa and their contribution to chloroplast phylogeny and adaptive evolution. International Journal of Molecular Sciences 19: 1847.

Yamamoto, Y., T. Majima, I. Saiki and T. Tani. 2003. Pharmaceutical evaluation of Glycyrrhiza uralensis roots cultivated in eastern Nei-Meng-Gu of China. Biological and Pharmaceutical Bulletin 26: 1144-1149.

Yang, J., K. Takayama, J.-S. Youn, J.-H. Pak and S.-C. Kim. 2020. Plastome characterization and phylogenomics of East Asian beeches with a special emphasis on Fagus multinervis on Ulleung Island, Korea. Genes 11: 1338.

Zerbino, D. R. and E. Birney. 2008. Velvet: Algorithms for de novo short read assembly using de Bruijn graphs. Genome Research 18: 821-829.

Zhang, W., H. Wang, J. Dong, T. Zhang and H. Xiao. 2021. Comparative chloroplast genomes and phylogenetic analysis of Aquilegia. Applications in Plant Sciences 9: e11412.

Zhao, Q.-Y., Y. Wang, Y.-M. Kong, D. Luo, X. Li and P. Hao. 2011. Optimizing de novo transcriptome assembly from shortread RNA-Seq data: A comparative study. BMC Bioinformatics 12: S2. 